\title{
La semántica de la construcción dativa del español: algunas restricciones a la alternancia con complementos preposicionales ${ }^{1}$
}

\author{
Nicolás Saavedra Garretón* \\ Pontificia Universidad Católica de Chile
}

\begin{abstract}
Resumen
Este artículo muestra la existencia de restricciones semánticas de alternancia que plantean algunos límites a dos conceptos desarrollados en el contexto de los estudios contemporáneos sobre la interpretación de la construcción dativa del español: las nociones de relación integral (Romero Morales 2008) y de relación genética (Gutiérrez Ordóñez 1999). La primera se ocupa de las relaciones semánticas que legitiman esta construcción y la segunda busca explicar la determinación de su significado a partir de otras oraciones con complementos preposicionales. Las mencionadas limitaciones a estos conceptos se exponen a partir del análisis de ejemplos que incluyen verbos pertenecientes a dos clases de dativo: los de transferencia material y los de movimiento físico, según la tipología de Delbecque y Lamiroy (1996). Los resultados de estos análisis muestran que la adecuación descriptiva de estas relaciones puede quedar restringida por efecto de los requisitos léxicos del verbo en
\end{abstract}

1 Este artículo ha sido desarrollado en el marco del proyecto Fondecyt Iniciación $\mathrm{n}^{\circ} 11130516$, "La sintaxis del aspecto en español: una propuesta desde la teoría de principios y parámetros". Investigador responsable: Nicolás Saavedra Garretón.

* Para correspondencia, dirigirse a: Nicolás Saavedra Garretón (lsaavedrag@uc.cl), Facultad de Letras, Pontificia Universidad Católica de Chile, Santiago 7820436, Chile. 
combinación con el potencial semántico de la construcción dativa del español.

Palabras clave: construcción dativa, semántica del español, preposiciones, alternancias, significado verbal.

The semantics of the Spanish Dative Construction:

SOME RESTRICTIONS ON THE ALTERNATION WITH PREPOSITIONAL COMPLEMENTS

\begin{abstract}
This article exposes the existence of semantic restrictions to alternations in the Spanish dative which lay out some boundaries for two concepts developed in the context of contemporary studies about the interpretation of the Spanish dative construction: the notions of comprehensive relation (Romero Morales, 2008) and genetic relation (Gutiérrez Ordóñez, 1999). The first notion takes on the semantic relations that license these constructions and the second one tries to explain how its meaning is determined taking as a basis the meaning of other sentences with fully prepositional complements. The aforementioned limitations to these concepts are exposed basing the analysis on examples that include verbs belonging to two classes of the Spanish dative: the ones belonging to the class of material transference and the ones belonging to the class of physical movement, according to Delbecque y Lamiroy's (1996) typology. The results of these analyses show that the descriptive adequacy of these relations can be restricted by the lexical requisites of the verb in combination with the semantic potential of the dative construction in Spanish.
\end{abstract}

Keywords: dative construction, Spanish semantics, prepositions, alternations, verb meaning.

Recibido: 27/03/2016 Aceptado: 24/01/2017 


\section{INTRODUCCIÓN}

En el ámbito de los estudios contemporáneos sobre la semántica de la construcción dativa ${ }^{2}$ del español existen dos propuestas conceptuales de especial relevancia: las nociones de relación integral (Romero Morales, 2008) y de relación genética (Gutiérrez Ordóñez 1999). La importancia de estos conceptos radica en que ofrecen, respectivamente, una concepción de la contribución de los complementos preposicionales y los argumentos dativos al significado global de la construcción y un análisis del significado de la misma basado en una relación hipotética de significado con estructuras lingüísticas primarias. Así, tomadas en conjunto, estas nociones buscan dar cuenta de las restricciones de significado que legitiman la construcción con clítico dativo a partir de procesos de alternancia con oraciones con complemento preposicional. Dada la importancia de estas propuestas para la investigación actual sobre la semántica de esta construcción del español, conviene detenerse en ellas para estudiar sus limitaciones y su rango de aplicabilidad en relación con ejemplos que permiten cuestionar algunos de sus supuestos.

La sección 2 establece una delimitación preliminar relativa a la selección de datos que serán estudiados en la sección 4 y las técnicas analíticas que se utilizarán. En la sección 3 se presentan los tres desarrollos teóricos que constituyen el trasfondo de este trabajo: en la sección 3.1 se expone un resumen de la tipología de dativos del español elaborada en Delbecque y Lamiroy (1996), poniendo énfasis en la caracterización de las dos clases de dativo que serán consideradas en los análisis: los dativos de transferencia material y los dativos de movimiento físico (secciones 3.1.1 y 3.1.2, respectivamente). Luego, en las secciones 3.2 y 3.3 se presentan los conceptos de relación integral y relación genética y en la sección 3.4 se exponen los contrastes y las complementariedades que cabe establecer entre estas nociones. En la sección 4 se desarrollan los análisis centrales de este artículo, examinando primero algunas restricciones semánticas de alternancia en dativos de transferencia material (sección 4.1) y determinando luego (sección 4.1.1) las implicancias de estos análisis para la propuesta tipológica de Delbecque y Lamiroy (1996) y para las nociones de relación integral y relación genética. Seguidamente, en la sección 4.2, se estudiarán

\footnotetext{
2 Las denominaciones 'construcción dativa' y 'construcción con clítico dativo' se utilizarán con idéntica denotación a lo largo de este trabajo.
} 
algunas restricciones semánticas de alternancia en dativos de movimiento físico, para luego considerar, en la sección 4.1.2 y siempre en relación con esta clase de dativo, algunas implicancias paralelas a las de la sección 4.1.1. Finalmente, en la sección 5 se sintetizarán las conclusiones obtenidas a partir de los desarrollos precedentes y se formularán proyecciones de investigación a partir de estas conclusiones.

\section{ALCANCE DE ESTE TRABAJO}

La cantidad y complejidad de los factores que intervienen en el estudio de la semántica de la construcción dativa del español imponen la elección de áreas específicas de estudio para un trabajo de esta extensión. Consecuentemente, este artículo se concentra en el análisis de algunos verbos y estructuras pertenecientes a dos subclases de construcciones dativas (transferencia material y movimiento físico, ver sección 3.1) dentro de la clase general de los dativos actanciales con estructuras transitivas de la tipología de Delbecque y Lamiroy (1996). Esto significa que las potenciales extensiones de nuestros resultados a otros tipos de dativos (e.g., dativos de transferencia verbal y perceptual, de movimiento abstracto, posesivos o dativos correspondientes tanto a estructuras intransitivas como no actanciales) no serán abordadas aquí. Otra estrategia de acotación corresponde a evitar la asunción a priori de algún modelo o teoría vigente (e.g., Teoría de Principios y Parámetros construccionista o lexicista, Gramáticas de Construcciones, Gramática Sistémico-Funcional o del Papel y la Referencia, entre muchas otras alternativas contemporáneas), dándole prioridad a métodos tradicionales de análisis gramatical y semántico (e.g., pruebas de alternancia, de conmutación, de obtención de contradicciones por negación, etc.). Esta corresponde, de hecho, a la orientación de la mayoría de las investigaciones contemporáneas sobre la construcción dativa del español que sirven de base para este trabajo, como puede apreciarse en los textos de Delbecque y Lamiroy (1996), Gutiérrez Ordóñez (1999) y Romero Morales (2008). Esto no significa asumir (también a priori) que cualquier modelo o teoría sería igualmente relevante en términos de situar estos análisis bajo sus supuestos particulares. Por el contrario, se dedicará una parte de la sección final de este artículo a indicar, precisamente, qué modelos o teorías podrían ofrecer mejores perspectivas en términos de establecer nuevas inferencias a partir de los resultados obtenidos. 


\section{CONCEPTOS TEÓRICOS PRINCIPALES: TIPOLOGÍA DE DATIVOS DEL ESPAÑOL, RELACIÓN INTEGRAL Y RELACIÓN GENÉTICA}

En las siguientes subsecciones se presentarán, de manera sucinta, los tres desarrollos que sirven de base para este trabajo: en primer lugar (sección 3.1), la propuesta tipológica de Delbecque y Lamiroy (1996) permitirá determinar las subclases específicas de dativos a las que pertenecen los verbos considerados en los análisis de las secciones 4.1 y 4.2. Luego, en las secciones 3.2 y 3.3 se presentarán, respectivamente, las nociones de relación integral y relación genética. Se trata de conceptos relevantes para este artículo en la medida en que caracterizan, en conjunto, las relaciones semánticoargumentales capaces de legitimar construcciones con clítico dativo en las que intervienen verbos de las clases seleccionadas en la sección 3.1.

\subsection{LA TIPOLOGÍA DE LOS DATIVOS DEL ESPAÑOL de Delbecque y Lamiroy (1996)}

En su trabajo de 1996, Delbecque y Lamiroy proponen una tipología de los dativos del español estructurada de la siguiente manera:

\begin{tabular}{|c|c|c|c|}
\hline \multirow[t]{11}{*}{ Dativos } & \multirow[t]{9}{*}{ Actanciales } & \multirow[t]{5}{*}{ Estructuras transitivas } & Clase I: Transferencia Material \\
\hline & & & $\begin{array}{l}\text { Clase II: Transferencia verbal y } \\
\text { perceptual }\end{array}$ \\
\hline & & & Clase III: Movimiento físico \\
\hline & & & Clase IV: Movimiento abstracto \\
\hline & & & Dativo posesivo \\
\hline & & \multirow[t]{4}{*}{ Estructuras intransitivas } & Interés \\
\hline & & & Movimiento físico \\
\hline & & & Movimiento psicológico \\
\hline & & & \begin{tabular}{|l|} 
Dativo posesivo \\
\end{tabular} \\
\hline & \multirow{2}{*}{\multicolumn{2}{|c|}{ No actanciales }} & En presencia de otro dativo \\
\hline & & & En ausencia de otro dativo \\
\hline
\end{tabular}

Para cada uno de los tipos más específicos de dativo de la última columna, las autoras ofrecen listas representativas de verbos, ejemplos de oraciones, 
una caracterización semántica esquemática, cada tipo y, finalmente, las propiedades distribucionales de estos mismos tipos (Delbecque y Lamiroy 1996: 88). A modo de ejemplo y sinopsis de las clases que serán consideradas en las secciones 4.1 y 4.2 , se presentan a continuación algunas de estas propiedades para los dativos de transferencia material y los dativos de movimiento físico.

\subsubsection{Dativos de transferencia material}

Lista representativa de verbos

confiar, devolver, enviar, infligir, otorgar, proporcionar, regalar, transferir, transmitir, entre otros (Delbecque y Lamiroy 1996: 91).

Ejemplos de oraciones

(1) a. Eva le dio un libro a Pepe.

b. Los traidores entregaron a los presos al enemigo.

c. El dativo le da jaquecas a Eva.

d. Legó todos los objetos de arte al museo. (Delbecque y Lamiroy 1996: 91)

Caracterización semántica (estructura eventiva)

El referente del sujeto gramatical de la voz activa (Suj) hace que el referente del complemento directo (CD) entre en el dominio del complemento indirecto (CI) (Delbecque y Lamiroy 1996: 91). Las autoras señalan además que el verbo dar corresponde al prototipo de este grupo, en la medida en que puede analizarse directamente como 'hacer llegar'.

Propiedades distribucionales

Delbecque y Lamiroy describen estas propiedades a partir de la siguiente estructura:

(2) $\mathrm{Suj}[+$ ANIMADO $]+\mathrm{V}+\mathrm{CD}[-\mathrm{ANIMADO}]+\mathrm{a}+\mathrm{CI}$ [+ANIMADO $]$

\subsubsection{Dativos de movimiento físico}

Lista representativa de verbos

acercar, agregar, añadir, aportar, echar, llevar, tirar, poner, entre otros (Delbecque y Lamiroy 1996: 94). 
Ejemplos de oraciones

(3) a. Llevaron a Juan al médico.

b. A Luisa le pusieron una inyección.

c. A la salsa le agregamos un poco de coñac.

d. Juan le aportó mucho a la empresa. (Delbecque y Lamiroy 1996: 94)

Caracterización semántica (estructura eventiva)

El referente del Suj hace que el referente del CD se mueva para llevarlo al dominio del referente del CI. El verbo prototípico de esta clase correspondería a llevar (Delbecque y Lamiroy 1996: 93).

Propiedades distribucionales

(4) $\operatorname{Suj}[+$ ANIMADO $]+v+(a)+C D[ \pm$ ANIMADO $]+C I[ \pm$ ANIMADO $]$

(Delbecque y Lamiroy 1996: 94)

Las propiedades semánticas y distribucionales de estas distintas clases de argumento dativo no poseen un carácter absoluto. Se trata, antes bien, de las características no marcadas de cada tipo, susceptibles de verse modificadas por extensión metafórica o por factores léxicos (Delbecque y Lamiroy 1996: 88). Además, las autoras asumen que la polisemia verbal puede producir efectos de transcategorización entre las distintas clases, como en el caso del verbo comunicar, que clasifican como dativo de transferencia material y perceptual (clase II) en Juan me comunicó que el congreso no tendría lugar y como dativo de movimiento (clase IV) en Juan comunicó su entusiasmo a todo el grupo (Delbecque y Lamiroy 1996: 88).

\subsection{El CONCEPTO DE RELACIÓN INTEGRAL: ROMERo MORALES (2008)}

Para Romero Morales (2008) las relaciones integrales corresponden a restricciones de significado que "condicionan la posibilidad de formar una construcción dativa" (2008: 44). Estas relaciones son de carácter semántico (e.g., relaciones parte-todo, de posesión, continente-contenido y figurafondo), se establecen entre el complemento directo y el complemento indirecto y se verifican a partir de dos pruebas de alternancia: (i) cuando una oración con complemento preposicional (ejemplos (a) en (6)-(8) abajo) puede alternar con una oración con clítico y complemento dativo (ejemplos (b) en (6)-(8)), entonces existe relación integral entre los complementos de estas oraciones y la construcción dativa es posible; (ii) cuando la construcción 
con complemento preposicional puede alternar con una estructura con tener como núcleo verbal (ejemplos (c) en (6)-(8)), entonces existe relación integral y la construcción dativa es, nuevamente, posible.

Relación integral parte-todo

(5) a. Puso una pistola en el cajón.

b. *Le puso una pistola al cajón.

c. *El cajón tiene una pistola. (Romero Morales 2008: 48)

Relación integral de posesión

(6) a. María envió una carta a Sara.

b. María le envió una carta a Sara.

c. María tiene la carta. (Romero Morales 2008: 46-47)

Relación integral continente-contenido

(7) a. Cargó la leña en el camión.

b. Le cargó la leña al camión.

c. El camión tiene leña. (Romero Morales 2008: 48)

Relación integral figura-fondo

(8) a. Espolvoreó chocolate en el pastel ${ }^{3}$.

b. Le espolvoreó chocolate al pastel.

c. El pastel tiene chocolate. (Romero Morales 2008: 49)

En conjunto, las dos pruebas señaladas permiten concluir que en (5) la relación entre los complementos no es integral, pues no existe alternancia con una construcción con clítico dativo (5b) ni tampoco con una construcción con tener (5c). Esta circunstancia apunta al modo en que Romero Morales concibe el carácter integral de estas relaciones:

3 Un evaluador anónimo indica el "carácter marginal o colocacional de ciertos usos" de verbos como espolvorear o jugar. No obstante, las (eventuales) regularidades cuantitativas de coocurrencia de estos verbos junto a otros lexemas o la posible escasez de sus instanciaciones estadísticas no parecen influir en su comportamiento relativo a pruebas como la de (8). 
"No basta (...) que haya un par de libros en el camión o un poco de chocolate espolvoreado en el suelo: el camión debe estar lleno de libros y el suelo de chocolate para que la relación se considere completa y pueda manifestarse sintácticamente por medio de una construcción dativa" (Romero Morales 2008: 49).

Así, los asteriscos de los ejemplos (5b) y (5c) indican una irregularidad semántica: tanto (5b) como (5c) son consideradas anómalas por Romero Morales porque la pistola no forma parte integral del cajón. Sin embargo, este autor indica que bajo un contexto apropiado la oración podría ser perfectamente interpretable, como en una película en donde la pistola está integrada en el cajón (Ibíd.: 48) y, por lo mismo, se establece una relación meronímica en donde el referente del complemento directo corresponde a una "parte posible" del complemento indirecto (Romero Morales 2008: 48).

\subsection{El CONCEPto de RElación GenétiCA: GutiérRez OrdóÑez (1999)}

En su trabajo de 1999 sobre los dativos, Gutiérrez Ordóñez toma como base la distinción entre complementos indirectos argumentales y complementos indirectos no argumentales para plantear la interrogante acerca de cómo se puede llegar a interpretar semánticamente el complemento dativo no argumental. Para el caso de los complementos indirectos argumentales, su interpretación semántica se determinaría claramente a partir de la entrada léxica del verbo. Así, en la entrada léxica de un verbo de transferencia material (ver sección 3.1.1) como enviar se especificaría, mínimamente, el número de complementos con que este verbo se combina, la función sintáctica de estos complementos (e.g., sujeto, complemento directo y complemento indirecto) y sus papeles o funciones semánticas (e.g., 'agente', 'tema' y 'meta', respectivamente) (Gutiérrez Ordóñez 1999: 1904). Tanto en construcciones con clítico dativo como en aquellas que carecen de este pronombre átono, la interpretación semántica del complemento indirecto será constante e inambigua, pues se estaría asignando léxicamente. Evidentemente, este modo de determinación léxica de la función semántica no está disponible para los complementos indirectos no argumentales. El mecanismo que operaría en este caso es definido por el autor en el siguiente párrafo:

En el caso de los $\mathrm{CInd}^{2}$ [complementos indirectos no argumentales] nos encontramos con un doble desvalimiento expresivo. Por un lado, al no ser valenciales, la presencia del verbo nada nos dice sobre la función semántica que desempeñan. Por otra, no adoptan una preposición plena. 
Vienen introducidos por el índice funcional más neutro y vacío de cuantos posee la lengua: la preposición $a$. Si no disponemos de rasgos que nos lo manifiesten, ¿cómo obtenemos los valores 'posesivo', 'iudicantis', 'dirección', 'contrafactivo', 'locativo'...que se asignan tradicionalmente a los dativos? Se debe a un proceso de inferencia, un proceso pragmático en el que se elige la interpretación más relevante para cada caso. Existe, no obstante, un dato lingüístico que encamina este proceso deductivo: la relación genética que mantienen todos estos dativos incorporados con las secuencias de origen. En ellas el sentido era más explícito al disponer de preposiciones plenas que lo hacían patente (Gutiérrez Ordóñez 1999: 1904).

Un poco más adelante, el autor refuerza esta idea de la siguiente manera:

“Los tradicionales dativos 'simpatéticos', 'iudicantis', 'commodi et incommodi', 'de dirección', 'final'...no son sino complementos indirectos incorporados que apoyan su denominación no en la estructura de dativo (que es única y común) sino en la paráfrasis (más o menos imperfecta) con la que cada decurso mantiene relación” (Gutiérrez Ordóñez 1999: 1905).

En definitiva, para Gutiérrez Ordóñez, la interpretación semántica de los complementos indirectos no argumentales de los ejemplos (9a-c) se obtendría (al menos parcialmente) a partir de la relación genética de alternancia que estos complementos mantendrían con los sintagmas preposicionales de los ejemplos $\left(9 \mathrm{a}^{\prime}-\mathrm{c}^{\prime}\right)$ :

(9) a. Le limpió las manos al niño. (Posesivo)

a’. Limpió las manos del niño.

b. Le puso orégano a la pizza. (Locativo)

b'. Puso orégano en la pizza.

c. Le jugó un partido a Costa. (Contrafactivo)

c’. Jugó un partido contra Costa. (Gutiérrez Ordóñez 1999: 1904-1905)

Lo esencial en estos pares de ejemplos, más allá del modo exacto en que Gutiérrez Ordóñez pueda definir valores semánticos tales como como posesivo, locativo y contrafactivo (pues dichos valores se asumen como conceptos básicos no definidos en el trabajo citado de este autor), corresponde al hecho de que se otorga un rol decisivo a las preposiciones de (9a'-c') en la asignación de estas mismas funciones semánticas a los complementos indirectos de las oraciones con clítico. 


\subsection{Semejanzas y CONTRASTES ENTRE LOS CONCEPTOS} DE RELACIÓN INTEGRAL Y RELACIÓN GENÉTICA

La característica común más evidente entre estas formulaciones corresponde a la prueba de alternancia entre estructuras con complemento preposicional y construcciones con clítico dativo, utilizada en ambos casos. Sin embargo, como se trata de conceptos que buscan responder a preguntas aparentemente distintas sobre la semántica de la construcción dativa del español, el uso de esta prueba podría ser, consecuentemente, diferente: en el caso de la propuesta de Romero Morales (2008), la interrogante que motiva el uso de la prueba de alternancia corresponde a la siguiente: ¿Cómo se legitima semánticamente la construcción con clítico dativo? Para el concepto de relación genética la pregunta corresponde a: ¿Cómo se obtiene la interpretación de la construcción con clítico dativo cuando el complemento indirecto es de naturaleza no argumental? Es claro que el uso de una misma prueba para intentar responder estas dos interrogantes justifica su puesta en relación. Se trata de un vínculo fácil de establecer, pues una construcción dativa es semánticamente interpretable en la medida en que existen factores de significado que legitiman su interpretabilidad. Desde el punto de vista del trabajo de Romero Morales (2008) estos factores corresponden, por supuesto, a las relaciones integrales. Existe, no obstante, un punto de contraste entre los conceptos en juego que atañe al estatus argumental del complemento indirecto: mientras la propuesta de Gutiérrez Ordóñez está pensada para explicar la interpretación de complementos indirectos no argumentales, el concepto de relación integral de Romero Morales parece ser relevante para complementos indirectos argumentales. Este última afirmación se justifica a partir de la elección de verbos que lleva a cabo este autor en sus ejemplos: entregar, enviar, poner, colocar, etc. Se trata, como puede observarse, de verbos que, o bien están incluidos en algún subtipo de la clase de las dativos argumentales con complemento directo de Delbecque y Lamiroy (1996, ver sección 3.1) o bien, podrían asignarse coherentemente a alguno de estos subtipos (e.g., la clase III para espolvorear). Sin embargo, la nitidez de este contraste debe ser tomada con precaución debido al hecho de que la clasificación de estos verbos como argumentales o no argumentales no siempre es consistente. En particular, el presente trabajo abordará el análisis de verbos locativos como poner (ver sección 4.2), que es categorizado dentro de la clase de los verbos que seleccionan dativo argumental por Delbecque y Lamiroy (1996: 94) y Romero Morales (2008: 25) pero en Gutiérrez Ordóñez (1999: 1876) es considerado tanto dentro de esta misma clase, como igualmente dentro de los ejemplos de verbos que participan en relaciones genéticas con construcciones de complemento preposicional 
(es decir, verbos que no seleccionan un dativo argumental) (Ibíd.: 1904). A nuestro juicio, más que constituir un mero error, esta doble categorización refleja un hecho al que Romero Morales apunta claramente en su trabajo:

Los constituyentes que expresan una locación (...) tradicionalmente no han sido considerados complementos indirectos. Cuando se ha querido subrayar su pertenencia a la valencia semántica del verbo se los ha tratado de manera específica como complementos locativos. Por el contrario, cuando el análisis se ha centrado en su interpretación semántica dentro de la oración, se han agrupado con los otros complementos circunstanciales. Pedimos disculpas al lector por tanta inexactitud, pero la, por así decirlo, transversalidad de la construcción dativa dentro del sistema lingüístico hace que ajuste mal dentro de las taxonomías lingüísticas con las que normalmente se caracteriza la lengua española (Romero Morales 2008: 23).

La complejidad a la que apunta Romero Morales permite poner en suspenso el carácter no argumental que, de acuerdo con Gutiérrez Ordóñez, poseen, al menos, algunas de las construcciones dativas que participan en las alternancias de la relación genética. Este hecho acerca aún más las relaciones semánticas propuestas por estos autores, y lo que esta aproximación sugiere es un mismo fenómeno visto desde dos puntos de vista: mientras la propuesta de Gutiérrez Ordóñez busca describir el fundamento semántico parcial de ciertas interpretaciones sistemáticas de las estructuras con clítico dativo, la formulación de Romero Morales clasifica semánticamente estas interpretaciones y las describe como factores de legitimación de la construcción dativa del español.

\section{RESTRICCIONES SEMÁNTICAS DE ALTERNANCIA EN DOS CLASES DE DATIVO DEL ESPAÑOL}

En esta sección principal analizaremos algunas restricciones de significado adicionales a las alternancias entre construcciones con complemento preposicional y construcciones con clítico dativo. Estas restricciones imponen limitaciones adicionales a las condiciones y patrones de alternancia correspondientes, respectivamente, a las relaciones integrales y genéticas.

Como ya se ha señalado en la introducción, el análisis se dividirá en dos partes: en la sección 4.1 se abordarán ejemplos en donde participan 
verbos de la Clase I (transferencia material) de la tipología de Delbecque y Lamiroy (1996) y en la sección 4.2 se considerarán ejemplos con verbos de la clase III (Movimiento físico) de esta misma tipología. La elección de estas dos clases se motiva en las siguientes consideraciones (más allá de los límites que la extensión de este trabajo impone): la clase de los verbos de transferencia material corresponde, para las autoras, a una suerte de prototipo alrededor del cual se organizan las clases II-IV. Esto se aprecia claramente por el hecho de que las clases II-IV son analizadas por estas autoras como extensiones de un esquema "básico" de transferencia representado por la clase I, la cual corresponde, por lo tanto, a una clase que ocupa un lugar de interés especial dentro de la tipología. En cuanto a la selección de la clase III, cabe señalar que ésta contiene verbos locativos que parecen sustraerse a los patrones de alternancia basados en relaciones integrales y genéticas. $\mathrm{Al}$ inicio de la sección 4.2 se presentan otras consideraciones que justifican la inclusión de esta clase de dativo.

Cabe señalar, finalmente, que en los análisis que siguen no se considerarán pruebas relativas a la alternancia con oraciones construidas con tener como núcleo verbal (e.g., los ejemplos (6c)-(8c)). La razón principal de esta omisión radica en que se trata de una prueba de aplicación limitada (e.g., la destrucción que el emperador le infligió a sus propios súbditos y *los súbditos tienen la destrucción) que parece estar sometida a condiciones distintas de las que se expondrán en la siguientes subsecciones. Por motivos de espacio, no será posible profundizar en las restricciones específicas relativas a esta prueba, que merecen ser tratadas en desarrollos aparte que profundicen, además, en el rango de significados de posesión ligados a la construcción dativa.

\subsection{RESTRICCIONES SEMÁNTICAS DE ALTERNANCIA EN ALGUNOS DATIVOS DE TRANSFERENCIA MATERIAL DEL ESPAÑOL}

Considérese el contraste de aceptabilidad ilustrado por el par de ejemplos de (10):
a. María envió una carta a Sara/a París.
b. *María le envió una carta a París. (Romero Morales 2008: 46-47)

Como verbo que selecciona un complemento indirecto en su valencia (es decir, léxicamente), enviar legitima complementos tanto animados como inanimados en (10a). La alternancia de estas oraciones con (10b) no es posible, sin embargo, de acuerdo al juicio de aceptabilidad de Romero 
Morales. Esto significa que los requerimientos léxicos del verbo no son suficientes para legitimar una estructura como (10b). La legitimación de esta estructura debe provenir, por lo tanto, de factores no léxicos. Una propuesta consistiría en asumir que la construcción con clítico dativo siempre selecciona un complemento dativo animado: $L e \mathrm{~V}$ SDet $a \mathrm{SDet}_{\mathrm{SN}_{+\mathrm{ANIMADO}}}{ }^{4}$ Es fácil probar, sin embargo, que este patrón no se cumple siempre: por ejemplo, con un verbo locativo como poner la construcción dativa acepta sin problema un complemento indirecto inanimado: Le puso el mantel a la mesa. En pocas palabras, el requisito de animacidad no pertenece a la construcción dativa (11a) sino a la combinación de la construcción dativa con un verbo como enviar (11b). Se trata de una combinación idiosincrásica, pues enviar sí puede seleccionar un complemento indirecto inanimado fuera de la construcción dativa, como se vio en (10a):

$$
\begin{aligned}
& \text { a. Le V SDet } a \mathrm{SDet} / \mathrm{SN} \pm_{\text {ANIMADO }} \\
& \text { b. Le enviar } \operatorname{Sdet} a \operatorname{SDet} / \mathrm{SN}_{+\mathrm{ANIMADO}}
\end{aligned}
$$

Existen, por tanto, restricciones de alternancia que pueden venir impuestas por la combinación de la construcción dativa con un verbo específico (o, por hipótesis, con una clase específica de verbos) y que también pueden involucrar preposiciones distintas de $a$, como puede observarse en los siguientes ejemplos:

a. Mi hermana en su boda regaló para las mujeres unas cajitas con unas velas y portavelas dentro muy chulas 5 .

b. Mi hermana le regaló a las mujeres una cajitas con unas velas.

c. La mamá de Sofía me comentaba en su comentario que hubo gente que le regaló para la pequeña Sofía cositas demasiado calurosas teniendo en cuenta que ella vive en Sevilla ${ }^{6}$.

El ejemplo (12b) muestra que el complemento indirecto de la construcción dativa también puede alternar con un complemento encabezado por para. Esta preposición, sin embargo, no equivale funcionalmente a la preposición

\footnotetext{
4 En donde SDet corresponde a 'Sintagma Determinante' y SN a 'Sintagma Nominal'.

5 El ejemplo (12a) ha sido extraído de http://forofemenino.hola.com/obsequios/queregalar-a-tus invitados/?PHPSESSID $=9 \mathrm{cb} 00 \mathrm{c} 1270 \mathrm{f} 44 \mathrm{fcdda} 6 \mathrm{~b} 6677 \mathrm{ca} 2 \mathrm{~b} 0512$. Recuperado el 19/11/16. El ejemplo (12b) corresponde a una modificación de (12a).

${ }_{6}$ Ejemplo extraído de: http://repolleteyprincesita.blogspot.cl/2012/09/regalar-sinpensar.html. Recuperado el 19/11/16.
} 
$a$, como se aprecia en el ejemplo (12c), en donde el sintagma encabezado por para no está en relación de concordancia con le (e.g., hubo gente que le regaló para las niñas cositas demasiado calurosas). Para Gutiérrez Ordóñez (1999), el valor semántico asociado al sintagma encabezado por para en (12a) y (12c) correspondería a 'beneficiario'. En relación a $a$, se puede adoptar la terminología de Romero Morales (2008) y considerar que el valor semántico corresponde a 'meta', "la persona o el lugar hacia el que se dirige la acción” (Romero Morales 2008: 11). Sin embargo, más allá de las definiciones nocionales que se asignen a estas funciones, lo relevante es notar que se trata de un conjunto de valores semánticos distintos que pueden alternar, igualmente, con la construcción con clítico dativo. En este conjunto es posible incluir, al menos, los valores correspondientes a para y $a$ :

\section{(13) Le V SDet $a$ SDet $/ \mathrm{SN}_{\{a \text { para }\}}$}

Podría sugerirse que, en realidad, el valor de $a$ es más general y de algún modo implica el valor de para. Sin embargo, es posible negar el complemento encabezado por para sin obtener una contradicción. No existe, por lo tanto, una relación de implicación de naturaleza semántica:

(14) Le regaló $a$ [las mujeres $]_{i, \text { meta }}$ una cajitas que no eran para ellas $_{\mathrm{i} \text {, beneficiario }}$

Otra alternativa, compatible con esta evidencia relativa a la negación de para, consistiría en proponer que, aunque no existe una relación de inclusión entre $a$ y para, sí existe una tercera función semántica más general que agrupa a ambas y es precisamente este valor general el que se asocia al complemento indirecto de la construcción dativa. Se trata de una solución que, de hecho, equivale formalmente a la estructura de (13). La diferencia, claro está, es que la representación del conjunto de valores de (13) es extensional y a lo que esta solución apunta es a formular una definición intensional de este conjunto. Sin embargo, del mismo modo en que una definición nocional exacta de los valores de a y para es mucho menos relevante que el simple hecho de comprender que estamos ante funciones semánticas distintas, la caracterización intensional del conjunto $\{a, p a r a\}$ es también secundaria frente el hecho de comprender que estos dos roles forman parte de un mismo conjunto y el complemento indirecto de la construcción dativa denota este conjunto ${ }^{7}$.

Nótese que esta solución implica, además, que la forma $a$ de la construcción sin clítico y la forma homófona de la construcción que posee clítico dativo no son funcionalmente 
Es posible, sin embargo, que la construcción dativa no siempre alterne con un complemento preposicional encabezado por para:

(15) a. Siendo testigo de la destrucción que el emperador infligió contra sus propios súbditos, Tatsumi se enfrenta a él ${ }^{8}(\ldots)$

b. (...) la destrucción que el emperador le infligió a sus propios súbditos (...)

c. ?(...) la destrucción que el emperador infligió para sus propios súbditos (...)

Estos ejemplos muestran que mientras la oración (15a) que posee un complemento preposicional encabezado por contra sí puede alternar con (15b), la alternancia de (15b) y (15c) no es posible. Aun en el caso en que se conciba alguna interpretación para (15c) (e.g., la destrucción que el emperador infligió (a SN/SDet) para (beneficio de) sus propios súbditos), el complemento encabezado por para no alterna con el complemento indirecto de la construcción con clítico dativo. En cambio, sí son posibles tanto el valor de $a$ (e.g., 'meta') como el valor de contra, que podría caracterizarse nocionalmente como 'maleficiario', correspondiente al referente que recibe los efectos negativos del evento denotado por infligir. Aparentemente, la posibilidad de asignar este valor de contra en la construcción con clítico dativo "bloquea" la asignación del valor de para, aun cuando esta última función forma parte del conjunto de valores semánticos denotados por el complemento indirecto (como se representa en la estructura de (13)). Dicho bloqueo, sin embargo, está motivado léxicamente, pues es concomitante con la aparición del verbo infligir:

$$
\text { Le infligir SDet } a \mathrm{SDet} / \mathrm{SN}_{\{a, \text { tara, contra\} }}
$$

El proceso opuesto se observa a partir de oraciones como las siguientes:

equivalentes. De este modo, el valor de la forma $a$ que forma parte del conjunto asociado al complemento dativo no coincide con el valor de la forma $a$ que encabeza gramaticalmente este mismo complemento en la construcción dativa. En Cuervo (2003: 38-39) y Demonte (1995: 6-7) es posible encontrar varios argumentos y ejemplos que respaldan esta diferencia funcional.

8 El ejemplo (15a) ha sido extraído de http://es.akamegakill.wikia.com/wiki/ Mata_al_Emperador. Recuperado el 19/11/16. Los ejemplos (15b) y (15c) corresponden a modificaciones de (15a). 
(17) a. Mi hermana le regaló a las mujeres una cajitas con unas velas.

b. *Mi hermana regaló contra las mujeres unas cajitas con unas velas.

La construcción dativa, en combinación con regalar alterna con estructuras que seleccionan los valores de $a$ y para excluyendo el valor de contra. De hecho, aun en ausencia del clítico, la combinación de regalar con un complemento encabezado por contra gatilla una secuencia anómala, como puede observarse en (17b). La estructura en este caso correspondería a la siguiente:

\section{Le regalar $\mathrm{SDet} a \mathrm{SDet} / \mathrm{SN}_{\{a, p a r a, e \text { entrtat }\}}$}

Alternativamente, este proceso podría concebirse no como un "bloqueo", sino como una equivalencia de funciones entre el verbo y la construcción:

$$
\text { Le regalar }{ }_{\{a, \text { para }\}} \text { SDet } a \text { SDet/SN } \operatorname{Sapara,contra\} }
$$

Desde el punto de vista de esta segunda alternativa, el valor de contra no puede actualizarse cuando la construcción dativa se combina con regalar porque no se trata de un valor que se encuentra en el conjunto seleccionado por el verbo. En términos conjuntistas ${ }^{9}$, esto equivale a afirmar que el complemento indirecto de la construcción dativa solo puede actualizar valores que pertenecen a la intersección del conjunto de valores del verbo y el conjunto de valores de la construcción ${ }^{10}$.

\subsubsection{Implicancias de los análisis de la sección 4.1 para la propuesta tipológica de Delbecque y Lamiroy (1996) y las relaciones integrales y genéticas}

El análisis del par de ejemplos de (10) muestra que la existencia de una relación integral de posesión entre el complemento directo e indirecto de

9 Cann (1993, a partir del capítulo 2) y el capítulo 1 de Partee et al. (1993) son dos introducciones muy útiles a la teoría de conjuntos, orientadas a sus aplicaciones lingüísticas.

10 No es posible ahondar aquí en una implicación potencialmente polémica de este análisis: la asunción de 'beneficiario’ y ‘maleficiario' como roles seleccionados léxicamente por el verbo (es decir, parte de la valencia semántica verbal). Una alternativa algo menos polémica a esta "expansión" de la valencia verbal podría asumir conjuntos jerarquizados de roles en donde solo algunos poseen carácter propiamente valencial. Se trata, sin embargo, de una discusión que no puede zanjarse dentro de los límites de este trabajo y que solo puede quedar planteada como un problema abierto de investigación. 
(10a) no es suficiente para legitimar su alternancia con la construcción con clítico dativo. En este caso, existe una restricción semántica adicional que corresponde a un requisito de animacidad producto de la combinación del verbo enviar con la construcción dativa. Esta restricción impide, correspondientemente, que la combinación de un verbo como enviar con un complemento preposicional con la forma en $\mathrm{SN} / \mathrm{SDet}_{\text {-ANIMADO }}$ establezca una relación genética con la construcción dativa que contribuya a la interpretabilidad de esta última.

En relación con la propuesta tipológica de Delbecque y Lamiroy (1996, ver sección 3.1), el requisito de animacidad señalado parece equivaler a lo representado por la estructura de las propiedades distribucionales de los dativos de transferencia material, que se reproduce nuevamente a continuación:

$$
\mathrm{Suj}[+ \text { ANIMADO }]+\mathrm{V}+\mathrm{CD}[-\mathrm{ANIMADO}]+\mathrm{a}+\mathrm{CI} \text { [+ANIMADO }]
$$

Sin embargo, esta representación no coincide exactamente con la estructura de (11a), ya que la restricción de animacidad aplica específicamente sobre la construcción con clítico dativo. En contraste, la estructura que proponen estas autoras parece apuntar más directamente a un requisito léxico de los verbos que pertenecen a la clase de enviar. Como ya se indicó, el presente análisis señala, en cambio, que la restricción de animacidad representada en (11b) no es una propiedad de la entrada léxica aislada ni tampoco de la construcción dativa, sino que se trata, antes bien, de una restricción específica de la combinación de esta construcción con enviar y, eventualmente, con la clase de los dativos de transferencia material, de acuerdo a la tipología de Delbecque y Lamiroy (1996).

En cuanto a los análisis sobre la alternancia de la construcción dativa con complementos preposicionales como para y contra (y siempre con núcleos verbales de transferencia material), ha sido posible especificar qué es lo que motiva la existencia de una interpretación común entre las oraciones que participan de esta alternancia (ejemplos de (12) y (15)): se trata de una coincidencia de significado entre algunos de los valores semánticos seleccionados por verbos como regalar e infligir y los valores correspondientes del complemento dativo. Interesantemente, esta propuesta permite cuestionar la necesidad de una noción como la de relación genética. Si la interpretación exitosa de la construcción con clítico está asegurada cuando la intersección de los valores semánticos del verbo y del complemento dativo no corresponde al conjunto vacío, entonces no es necesario postular la existencia de una relación genética entre dos estructuras: para que la construcción dativa sea interpretable, basta con que existan valores semánticos compartidos entre una unidad léxica verbal y esta construcción. 


\subsection{RESTRICCIONES SEMÁNTICAS DE ALTERNANCIA EN ALGUNOS DATIVOS DE MOVIMIENTO FÍSICO DEL ESPAÑOL}

En esta sección se analizan casos en los que se incluyen verbos pertenecientes la Clase III de la tipología de Delbecque y Lamiroy (1996). En particular, se considerarán ejemplos con verbos locativos como acercar, agregar y poner, que se analiza a continuación:

(21) a. Puso el mantel en la mesa.

b. Le puso el mantel a la mesa.

c. *Puso el sombrero en Sara.

d. Le puso el sombrero a Sara. (Romero Morales 2008: 26)

Romero Morales (2008: 25) explica el contraste de aceptabilidad en (21c) y (21d) asumiendo que los verbos locativos como poner o colocar solo aceptan locaciones animadas ${ }^{11}$ cuando aparecen en forma dativa. Esta explicación asume una correlación entre tres tipos de unidad lingüística: el verbo locativo con sus requisitos de selección semántica, el sintagma nominal en función de complemento dativo en conjunto con su rasgo semántico \pm ANIMADO, y la estructura específica de la construcción dativa. La explicación que Romero Morales ofrece para el contraste de (21c-d) puede representarse a partir de las siguientes estructuras:
a. Poner SDet en SDet/SN
b. Le poner $\mathrm{SDet} a \mathrm{SDet} / \mathrm{SN} \pm_{\text {ANIMADO }}$

Claramente (22a) contrasta con (22b) por la presencia de la preposición en y la consecuente ausencia del clítico dativo y la forma $a$. Sin embargo, la restricción al rasgo-ANIMADO del complemento indirecto no puede atribuirse exclusivamente a la preposición en, pues una oración como Pensó en Sara es perfectamente aceptable. El único constituyente que en (22a) podría codeterminar este requerimiento, en conjunto con la preposición, es el verbo locativo poner. Así, la estructura de (22a) puede ser interpretada

11 En estricto rigor, Romero Morales restringe este requisito a locaciones humanas (Romero Morales 2008: 25). Sin embargo, es claro que el juicio de aceptabilidad para el ejemplo (17a) se mantiene si 'Sara' denota una entidad animada no humana (e.g., una mascota). 
como un requisito léxico de poner expresable de modo condicional ${ }^{12}$ : si el complemento locativo de poner está encabezado por la preposición en, entonces el complemento de la preposición debe ser compatible con el rasgo -ANIMADO. El complemento de la preposición en (21c), Sara, posee el rasgo léxico +ANIMADO y, por lo tanto, no satisface este requisito. Evidentemente esta restricción no se presenta en la estructura con clítico dativo, pues de lo contrario una estructura como Le poner SDet $a$ SDet/ $\mathrm{SN}_{\text {-ANIMADO }}$ sería agramatical y claramente este no es el caso, como puede observarse en (21b). Así, el requisito -ANIMADO en el complemento indirecto corresponde a un rasgo de la estructura que se representa en (22a). ¿Se trata, sin embargo, de un contraste entre una unidad parcialmente composicional (representada en (22a), que tendría un cierto grado de fijación al adquirir una interpretación específica en combinación con poner) y otra plenamente composicional (representada en (22b))? La estructura de (23a) presenta el rasgo \pm ANIMADO como parte de la construcción con clítico dativo. Esta asunción permite un análisis al menos parcialmente composicional de la estructura de (23c), pues dicha estructura se compone a partir de la combinación del lexema poner en (23b) (que posee un rasgo categorial verbal V) con la estructura de (23a):
a. Le V SDet $a \mathrm{SDet} / \mathrm{SN} \pm_{\text {ANIMADO }}$
b. poner $_{\mathrm{v}}$
c. Le poner $\mathrm{SDet} a \mathrm{SDet} / \mathrm{SN} \pm_{\text {ANIMADO }}$

Asumiendo una estructura como (23a), es posible describir cómo se obtiene la interpretación de (21d): se trata de una estructura producida a partir de la combinación de (24a) y (24b) con la estructura (23a):
a. Poner $_{\mathrm{v}}$
b. $\operatorname{Sara}_{\mathrm{SN} / \text { ANIMADO }}$

En cuanto a (25a), se trata de una estructura producida a partir de la combinación de (25c) con (25e) y (25f). En tanto, (25b) corresponde a una estructura formada a partir de la combinación de (25d) con (23a), (25e) y (25f):

12 De modo similar a como se concibe el contenido de las unidades léxicas en el enfoque teórico de la Sintaxis Dinámica. Ver Cann, Kempson y Marten (2005) y Saavedra (2015). 
(25) a. Puso las cortinas en la habitación.

b. Le puso las cortinas a la habitación.

c. Poner SDet en $\mathrm{SDet} / \mathrm{SN}_{\text {-ANIMAdo }}$

d. poner $_{\mathrm{V}}$

e. [la habitación $]_{\text {SDet/-ANIMADO }}$

f. [las cortinas $]_{\text {SDet/-ANIMADO }}$

Bajo esta perspectiva analítica es relevante considerar un ejemplo como el de (26):

(26) Harry se quitó la chaqueta y envolvió el cadáver de Dobby ${ }^{13}$, Ron humildemente se quitó los zapatos y los calcetines y los puso en los pies de Dobby, y Dean puso un sombrero en Dobby, cubriendo su rostro ${ }^{14}(\mathrm{el}$ énfasis es del autor de este artículo).

En apariencia, este ejemplo estaría contradiciendo el juicio de aceptabilidad de Romero Morales en (21a). Sin embargo, esta aparente contradicción se resuelve a partir del rasgo -ANIMADO de Dobby en el contexto de (26). El nombre propio en (27a) es, por tanto, compatible con la estructura de (27b):
a. Dobby $y_{\text {SN/ANIMADO }}$
b. Poner SDet en $\mathrm{SDet} / \mathrm{SN}_{\text {-ANIMAdo }}$

La posibilidad de alternancia con verbos locativos de la clase III no solo se da con la preposición en. En los ejemplos que se muestran a continuación pueden observarse complementos encabezados con hasta y sobre: ${ }^{15}$

(28) a. El hombre acercó los equinos hasta ellos y luego los montaron ${ }^{15}$.

b. El hombre les acercó los equinos a ellos (...)

13 En el mundo ficcional de las novelas de Harry Potter, Dobby es un elfo y, por lo tanto, una entidad animada. Ver, por ejemplo, http://es.harrypotter.wikia.com/wiki/Dobby.

14 Ejemplo extraído de http://es.harrypotter.wikia.com/wiki/Funeral_de_Dobby. Recuperado el 20/3/16.

15 Ejemplo extraído de https://books.google.cl/books?id=GCGYBAAAQBAJ\&lpg=PT6 $1 \& \mathrm{dq}=\mathrm{Die} \% 20 \mathrm{Ce} \% \mathrm{C} 3 \% \mathrm{~B} 1 \% \mathrm{C} 3 \% \mathrm{BFa} \% 3 \mathrm{~A} \% 20 \mathrm{El} \% 20 \mathrm{fin} \% 20 \mathrm{del} \% 20$ reino $\% 20 \mathrm{del} \% 20 \mathrm{Sol} \&$ $\mathrm{hl}=$ es\&pg $=\mathrm{PT} 61 \mathrm{H}=$ =onepage $\& \mathrm{q}=\mathrm{Die} \% 20 \mathrm{Ce} \% \mathrm{C} 3 \% \mathrm{~B} 1 \% \mathrm{C} 3 \% \mathrm{BFa}: \% 20 \mathrm{El} \% 20 \mathrm{fin} \% 20 \mathrm{del} \% 20$ reino\%20del $\% 20$ Sol\&f=false. Recuperado el 19/11/16. El ejemplo (28b) corresponde a una modificación de (28a). 
c. Tres vecinos nos muestran la deco de sus casas. Se agregó una placa de zinc sobre la mesa de la cocina ${ }^{16}$.

d. Se le agregó una placa de zinc a la mesa de la cocina.

Estos ejemplos muestran un fenómeno similar al que se analizó en relación con los verbos infligir y regalar: la construcción con clítico dativo le asigna un valor semántico general al complemento indirecto, en relación con la cual los valores de significado de en, hasta, y sobre son más específicos. La consecuencia de esto es clara: el significado asociado al complemento indirecto de la construcción dativa subespecifica los valores semánticos más determinados de las tres preposiciones mencionadas y presentes en las oraciones con complemento preposicional. Al igual que en (13), podemos representar este valor general del complemento dativo como un conjunto que incluye todos los valores semánticos específicos de las preposiciones: ${ }^{16}$

$$
\text { Le V SDet } a \mathrm{SDet} / \mathrm{SN}_{\{e n, \text { sobre,hasta }\}}
$$

Relaciones de exclusión similares a las observadas en el caso de contra y para se pueden producir también aquí. Un ejemplo evidente corresponde al de los valores opuestos de las preposiciones sobre y bajo. Nótese que ambas pueden alternar con la construcción dativa:

(30) a. Se agregó una placa de zinc sobre la mesa de la cocina.

b. Se agregó una placa de zinc bajo la mesa de la cocina.

c. Se le agregó una placa de zinc a la mesa de la cocina.

Un efecto de selección entre valores semánticos preposicionales mutuamente excluyentes se observa a partir del verbo locativo superponer, perteneciente también a la clase III de la tipología de Delbecque y Lamiroy (1996: 94): ${ }^{17}$

(31) a. Con ayuda de la informática, Schwartz superpuso el autorretrato de Leonardo sobre el lienzo de La Gioconda ${ }^{17}$.

16 Ejemplo extraído de https://es.pinterest.com/pin/551198441876382742/. Recuperado el 19/11/16. El ejemplo (28d) corresponde a una modificación de (28c).

17 Ejemplo extraído de http://blog.tusmedios.es/la-selfi-de-robinson/. Recuperado el 20/3/16. Recuperado el 19/11/16. Los ejemplos (31b) y (31c) corresponden a modificaciones de (31a). 
b. Schwartz le superpuso el autorretrato de Leonardo al lienzo de La Gioconda.

c. *Schwartz superpuso el autorretrato de Leonardo bajo el lienzo de La Gioconda.

En estos ejemplos se observa que la construcción dativa de (31a) solo puede alternar con (31c), a diferencia del ejemplo (30c), que podía alternar con sobre o bajo. Se trata de una restricción léxicamente gatillada por el verbo superponer en combinación con la construcción dativa:

$$
\text { Le superponer }{ }_{\{\text {sobre }\}} \text { SDet } a \text { SDet } / \mathrm{SN}_{\{s o b r e, b a j o\}}
$$

La restricción interpretativa puede representarse nuevamente a partir de una restricción de intersección de valores: el valor semántico correspondiente a la interpretación del complemento dativo debe pertenecer a la intersección del conjunto de valores asociado a este complemento con el conjunto de valores seleccionados por el verbo. Por lo tanto, esta restricción excluye el valor de bajo como valor posible del complemento dativo en (31b).

\subsubsection{Implicancias de los análisis de la sección 4.2 para la propuesta} tipológica de Delbecque y Lamiroy y las relaciones integrales y genéticas

El contraste de aceptabilidad de los ejemplos (21c-d) impone una clara interrogante a las nociones de relación integral y relación genética: ¿cómo es posible que la construcción con complemento preposicional de (21c) sea anómala pero la estructura con clítico dativo de (21d) con constituyentes léxicos equivalentes a los de (21c) plenamente aceptable? Lo que impide que se produzca una equivalencia interpretativa entre ambas estructuras corresponde a una restricción de animacidad que, a diferencia de los ejemplos (10a-b), se establece en este caso en la estructura con complemento preposicional (21c). Esto muestra, nuevamente, que existen condiciones específicas introducidas por la combinación de un verbo particular (o, potencialmente, de una clase verbal particular), como es el caso de poner, con un complemento preposicional encabezado por una preposición como en. Esta restricción impide que una relación interpretativa como la que Gutiérrez Ordóñez (1999) propone pueda legitimar la alternancia de (21c) con (21d). Es interesante notar que la estructura propuesta por Delbecque y Lamiroy (1996) para representar las propiedades distribucionales de los verbos de las Clase III tampoco captura la restricción de (22a):

$$
\text { Suj[+ANIMADO }]+v+(a)+C D[ \pm A N I M A D O]+C I[ \pm \text { ANIMADO }]
$$


Esto es esperable si se asume que (33) representa la estructura defectiva de los verbos de esta clase, sin llegar a incluir condiciones combinatorias que no surgen de ningún constituyente léxico verbal ni preposicional aislado. Así, más que a una limitación de la propuesta de Delbecque y Lamiroy (1996), este hecho señala la necesidad de determinar qué condiciones semánticas más específicas operan dentro de las clases verbales de la tipología de estas autoras.

\section{DISCUSIÓN FINAL Y PROYECCIONES DE ESTE TRABAJO}

A modo de resumen de los resultados de este trabajo, cabe señalar, en primer lugar, que la legitimación semántica de la construcción dativa puede estar sometida a restricciones más particulares que las impuestas por las relaciones integrales. Dichas condiciones pueden surgir de la combinación de verbos específicos con la construcción, como es el caso de enviar y la restricción de animacidad que aparece al combinar este verbo con la construcción con clítico dativo. El mismo tipo de fenómeno fue observado en la sección 4.2, en el análisis basado en los ejemplos de (21). Nuevamente, se trata de un caso en que la combinación de un verbo (poner) con un constituyente complejo (un sintagma preposicional encabezado por en) impone restricciones de animacidad que restringen la interpretabilidad de la alternancia con la construcción dativa.

En segundo lugar, es importante enfatizar que tras el análisis de ejemplos como los de (12), (15) y (17) (sección 4.1) y (28),(30) y (31) (sección 4.2), la evidencia es convergente con lo señalado en el párrafo anterior: la alternancia entre estructuras con complementos preposicionales (tales como como para, contra, sobre y bajo) y la construcción dativa está condicionada por el potencial semántico combinatorio de los verbos y de la construcción.

Estos resultados permiten también relativizar la amplitud de aplicación del concepto de relación genética, pues no ha sido necesario postular un significado primario (asociado a una oración con complemento preposicional) para mostrar cómo el argumento dativo puede llegar a poseer una interpretación particular. Esta interpretación, por el contrario, puede obtenerse aisladamente a partir de los valores semánticos preposicionales denotados por el complemento encabezado por la forma $a$ de la construcción dativa y los valores equivalentes seleccionados por los verbos incluidos en los análisis. 
En cuanto a las proyecciones de este trabajo, existen, al menos, dos líneas productivas de avance: en primer lugar, será importante aplicar las pruebas de alternancia de este artículo a un mayor número de verbos de las clases I y III de la tipología de Delbecque y Lamiroy (1996). Esto permitiría establecer si se trata de clases homogéneas en cuanto a los valores semánticos preposicionales denotados por el argumento dativo o si, por el contrario, existen subclases que poseen valores distintos y mutuamente incompatibles. Las mismas interrogantes pueden plantearse en relación con un número mayor de clases de esta tipología. La segunda proyección de este trabajo tiene relación con los modelos y/o teorías particulares que podrían compatibilizar mejor con estos resultados para producir nuevas conclusiones (más particularizadas a las perspectivas que se adopten). Es claro que los modelos de corte construccionista ${ }^{18}$ poseerían una pertinencia particular, pues se ha mostrado que las alternancias consideradas pueden estar restringidas por factores no exclusivamente léxicos, sino producto de la combinación de unidades léxicas verbales particulares con el significado asociado a una construcción. Así, será relevante determinar qué enfoque construccionista contemporáneo se adecúa mejor a los análisis desarrollados en este trabajo.

18 Actualmente, estos modelos pueden provenir tanto del campo de la lingüística cognitiva, como es el caso de las Gramáticas de Construcciones (e.g., Gonzálvez-García (2012) y Goldberg (1995), entre otros) como desde la Teoría de Principios y Parámetros, con orientaciones como las de Borer (2005) y Ramchand (2008), entre otros. El punto común de estas perspectivas radica en la idea de que el significado lingüístico no es un fenómeno exclusivamente léxico sino también estructural: las construcciones lingüísticas también pueden poseer significado. Por lo tanto, la composicionalidad del significado lingüístico no puede reducirse a 'significado léxico más sistema computacional-combinatorio', como en enfoques estrictamente lexicistas (e.g., Chomsky (2016), Grimshaw (1990)). Por otra parte, el contraste entre estas dos aproximaciones construccionistas ha sido caracterizado por Ramchand (2008) de la siguiente manera: las Gramaticas de Construcciones asumen que existe una analogía entre las construcciones gramaticales y las unidades léxicas, pues tanto las primeras como las segundas deben ser memorizadas. Los enfoques 'generativo-constructivistas' (asumiendo la denominación de Ramchand (2008)), asumen, por el contrario, que las construcciones pueden llegar a tener significado "because they are systematically constructed as part of a generative system (syntactic form) that has predictable meaning correlates" (Ramchand 2008: 11). 


\section{REFERENCIAS BIBLIOGRÁFICAS}

Borer, Hagit. 2005. Structuring Sense Volume II: The Normal Course of Events. Oxford: Oxford University Press.

Bosque, Ignacio y Violeta Demonte (eds.). 1999. Gramática descriptiva de la lengua española. Madrid: Espasa Calpe.

Cann, Ronnie. 1993. Formal Semantics: An introduction. Cambridge: Cambridge University Press.

Cann, Ronnie; Ruth Kempson y Lutz Marten. 2005. The Dynamics of Language: An Introduction. Oxford: Elsevier.

Chosmky, Noam. 2016. The Minimalist Program (2da. Ed.). Cambridge Mass.: MIT Press.

Cuervo, María Cristina. 2003. Datives at large. Tesis para optar al grado de Doctora en Filosofía en Lingüística, MIT.

Delbecque, Nicole y Béatrice Lamiroy. 1996. Towards a typology of the Spanish dative. En William Van Belle y Willy Van Langendonck (eds.), pp. 74-117.

Demonte, Violeta. 1995. Dative alternation in Spanish. Probus 7(1): 5-30.

Goldberg, Adele. 1995. Constructions: A Construction Grammar Approach to Argument Structure. Chicago: Chicago University Press.

Gonzálvez-García, Francisco. 2012. La(s) Gramática(s) de Construcciones. En Iraide Ibarretxe-Antuñano y Javier Valenzuela (dirs.), pp. 249-280.

Grimshaw, Jane. 1990. Argument Structure. Cambridge Mass.: MIT Press.

Gutiérrez OrdóÑez, Salvador. 1999. Los dativos. En Ignacio Bosque y Violeta Demonte (eds.), pp.1855-1930.

Ibarretxe-Antuñano, Iraide y Javier Valenzuela (dirs.). 2012. Lingüística Cognitiva. Barcelona: Anthropos.

Partee, Barbara; ter Meulen, Alice y Robert Wall. 1993. Mathematical Methods in Linguistics. Dordrecht: Kluwer.

Ramchand, Gillian. 2008. Verb Meaning and the Lexicon: A First-Phase Syntax. Cambridge: Cambridge University Press.

Romero Morales, Juan. 2008. Los dativos en el español. Madrid: Arco.

SaAVEdra, Nicolás. 2015. Sintaxis Dinámica: un modelo de procesamiento sintácticosemántico. Logos: Revista de Lingüistica, Filosofía y Literatura 25(1): 87-97.

Van Belle, William y Willy van Langendonck (eds.). 1996. The Dative Volume 1: Descriptive Studies. Amsterdam: John Benjamins. 